Blob Dynamics in 3D BOUT Simulations of Tokamak Edge Turbulence

D.A. Russell, D.A. D'Ippolito, J.R. Myra, W.M. Nevins, X.Q. Xu

August 24, 2004

Physical Review Letter 
This document was prepared as an account of work sponsored by an agency of the United States Government. Neither the United States Government nor the University of California nor any of their employees, makes any warranty, express or implied, or assumes any legal liability or responsibility for the accuracy, completeness, or usefulness of any information, apparatus, product, or process disclosed, or represents that its use would not infringe privately owned rights. Reference herein to any specific commercial product, process, or service by trade name, trademark, manufacturer, or otherwise, does not necessarily constitute or imply its endorsement, recommendation, or favoring by the United States Government or the University of California. The views and opinions of authors expressed herein do not necessarily state or reflect those of the United States Government or the University of California, and shall not be used for advertising or product endorsement purposes. 


\title{
Blob Dynamics in 3D BOUT Simulations of Tokamak Edge Turbulence*
}

\author{
D. A. Russell, ${ }^{1}$ D. A. D'Ippolito, ${ }^{1}$ J. R. Myra, ${ }^{1}$ W. M. Nevins ${ }^{2}$ and X. Q. Xu ${ }^{2}$ \\ ${ }^{1}$ Lodestar Research Corporation, Boulder CO 80301 \\ ${ }^{2}$ Lawrence Livermore National Laboratory, Livermore CA 94551
}

\begin{abstract}
Propagating filaments of enhanced plasma density, or blobs, observed in 3D numerical simulations of a diverted, neutral-fueled tokamak are studied. Fluctuations of vorticity, electrical potential $\phi$, temperature $\mathrm{T}_{\mathrm{e}}$ and current density $\mathrm{J}_{\|}$associated with the blobs have a dipole structure perpendicular to the magnetic field and propagate radially with large $\mathbf{E} \times \mathbf{B}$ drift velocities $(>1 \mathrm{~km} / \mathrm{s})$. The simulation results are consistent with a 3D blob dynamics model that incorporates increased parallel plasma resistivity (from neutral cooling of the X-point region), blob disconnection from the divertor sheath, X-point closure of the current loops, and collisional physics to sustain the $\phi, \mathrm{T}_{\mathrm{e}}, \mathrm{J}_{\|}$dipoles.
\end{abstract}

PACS numbers: 52.25.Fi, 52.35.Ra, 52.55.Fa, 52.65.-y

In magnetized plasma, balancing the Lorentz force against a force $\mathbf{F}$ that is independent of charge gives rise to a polarizing drift: electrons and ions drift in opposite directions, perpendicular to the force and to the magnetic field B. By this mechanism isolated monopole concentrations of plasma density (referred to as "blobs") 1,2 become charge polarized and acquire an electric field $\mathbf{E}$ in the $\mathbf{F} \times \mathbf{B}$ direction. The resulting (charge independent) " $\mathbf{E} \times \mathbf{B}$ " drift propels the density blob as a whole in the direction of $\mathbf{F}$, giving rise to coherent objects that are analogous to vortices in hydrodynamic flow.

In plasma experiments ${ }^{3}$ blobs have been observed as filaments along the field line but localized in the twed imensional (2D) plane $\perp \mathbf{B}$. In tokamaks, the force $\mathbf{F}$ arises from the centripetal acceleration of the fluid plasma as it streams along the curved magnetic field lines of the torus. The resulting $\mathbf{E} \times \mathbf{B}$ drift is radially outward at the outboard midplane where curvature-driven instabilities thrive and the associated turbulence seeds blob formation (as shown in the present paper). Krasheninnikov suggested this mechanism ${ }^{1}$ to explain the highly intermittent, convective density transport observed in the open-field-line "scrape-off-layer" (SOL) of many plasma experiments. It has also been invoked in the context of pellet deposition. ${ }^{4}$ 
A requirement for significant blob radial transport is that parallel currents $\mathrm{J}_{\|}$do not drain away the induced charges on the convective timescale. In the $\mathrm{SOL}, \mathrm{J}_{\|}$is hindered by "sheath resistivity" for $2 \mathrm{D}$ blobs, which are constant along $\mathbf{B}$ and therefore connect to the divertor sheaths. The sheath sets the blob voltage-current relation ${ }^{1,2} \mathrm{~J}_{\|}=\alpha \phi$ with sheath conductivity parameter $\alpha \sim n \mathrm{e}^{2} \mathrm{c}_{\mathrm{S}} / \mathrm{T}_{\mathrm{e}}$ where $\mathrm{c}_{\mathrm{S}}=\left(\mathrm{T}_{\mathrm{e}} / \mathrm{m}_{\mathrm{i}}\right)^{1 / 2}$ is the sound speed. The $2 \mathrm{D}$ model thus determines the potential $\phi$ and the radial blob velocity. Previous work ${ }^{5}$ shows that the model agrees with some aspects of experiments and simulations.

This theoretical work, and the extensive experimental data ${ }^{3}$ which motivated it, suggests that SOL particle transport, and sometimes even energy transport, are dominated by blobs. Although blobs are born intermittently under conditions of strong turbulence near the magnetic separatrix, radial blob motion across the SOL is simple (ballistic) and is amenable to reduced modeling.

Here, we present the first study of blobs generated in 3D fluid turbulence simulations 6 for a diverted tokamak. We will show that (i) blobs are produced near the separatrix by the background turbulence and convect outwards; (ii) the blob's internal structure and radial velocity differs from the predictions of sheath-connected 2D models; and (iii) the blob dynamics is qualitatively consistent with a generalized 3D model which includes the effects of X-point geometry on the plasma current path.

The simulations were carried out using the BOUT turbulence code, ${ }^{7}$ which employs Braginskii fluid equations and realistic 3D tokamak magnetic field geometry (magnetic separatrix and X-points), and were fueled by neutral particle injection concentrated about the X-point in a diverted, DIII-D deuterium plasma. The neutral density profile $\mathrm{n}_{0}(\mathrm{x})$ decreases exponentially from the simulation boundary, $\mathrm{x}=3 \mathrm{~cm}$, to the separatrix, $\mathrm{x}=0$, on a spatial scale that depends on the local plasma temperature and density. ${ }^{8}$ [Here, $(\mathrm{x}, \mathrm{y}, \mathrm{z})$ denote the radial, poloidal, and parallel coordinates at the midplane; there are $(50,64,64)$ grid points, respectively, in the simulation.] Simulation results were analyzed using the gyrokinetic visualization (GKV) tools. ${ }^{9}$

The plasma density increases with time and eventually saturates in a state of strong turbulence characterized by order unity fluctuations, which can sometimes challenge the simulation as discussed later. The blob emission rate increases dramatically after the average density saturates $(\mathrm{t}>800 \mu \mathrm{s})$ at a value of $\langle\mathrm{n}\rangle=1.5 \mathrm{x}$ 
$10^{13} / \mathrm{cm}^{3}$ at the separatrix in the outboard midplane, where $\left\langle\mathrm{T}_{\mathrm{e}}\right\rangle=28 \mathrm{eV}$ and $\left\langle\Omega_{\mathrm{D}}\right\rangle=1.1 \times 10^{8} \mathrm{rad} / \mathrm{s} \quad\left(\mathrm{B}_{0}=2.3\right.$

T). (Here, “<>" indicates poloidal and time averaging for $t>800 \mu$ s.) Fig. 1 shows the strong correlation of density and vorticity $\omega_{\mathrm{z}} \propto \nabla^{2} \phi$ in the (y, t) plane for $\mathrm{x}=3 \mathrm{~cm}$ at these later times. At fixed times in the (x,y) plane (not shown), blobs are seen to have tails stretching back from the nose (leading edge) to the region of strong turbulent fluctuations where they are born, within about $1.5 \mathrm{~cm}$ of the separatrix. Despite this restricted radial domain, the blobs' internal structures, especially the dipole vorticity (see below), are clearly discerned once the blobs are freely propagating; we believe that if more of a radial inertial range were included in the simulation our conclusions would change little, if at all.

In Fig. 2, we focus on the properties of the circled blob in Fig. 1. In the inset we zoom in on the blob: line contours of ion density are superimposed on an intensity plot of charge density (vorticity) on the (y,t) plane. The charge separation is apparent, and the \pm charges pile up on the perpendicular "boundaries" of the blob density profile, $\mathrm{y} \approx \pm a$, respectively, where $a$ is the blob's perpendicular scale; a dipole charge density is consistently associated with isolated density maxima in the SOL of the simulations.

In the main body of Fig. 2 we plot the local maximum (+) and minimum (-) vorticities associated with the nose of the blob and the vorticity at the density maximum (0) versus radial position. Note that $\omega_{z}(0)$ has decayed away for this blob before $\omega_{z}( \pm)$ arises, consistent with the predicted reduction of charge separation by blob spin. ${ }^{10}$ Not all blobs examined demonstrate this simple decay; some central vorticities are much more erratic. Typically, a blob can be identified only after the dipole vorticity has emerged. (These blob trajectory records are constructed backwards in time: from $\mathrm{x}=3 \mathrm{~cm}$ where identification is easy, toward the separatrix, until the dipole is lost in turbulence.) The blob's coherence is related to the strength of the vorticity dipole. The blob is a coherent structure to the extent that its lifetime is longer than the transit time across one blob radius, which approximately equals its internal circulation time around each pole, $\tau_{\mathrm{C}}=2 \pi /\left|\omega_{( \pm)}\right| \sim a / \mathrm{v}_{\mathrm{x}}$. From Fig. $2 \tau_{\mathrm{C}}<3 \mu \mathrm{s}$, whereas the lifetime of the blob is limited in the simulation by the transit time across the finite radial domain, $\tau_{\mathrm{T}} \sim \Delta \mathrm{x} / \mathrm{v}_{\mathrm{x}} \sim 10 \mu \mathrm{s}$. Note from Fig. 2 that the analog of the usual eddy turnover time is $\tau_{\text {ed }}=2 \pi /\left|\omega_{(0)}\right| \sim 2 \pi /(3 \mathrm{x}$ $\left.10^{6} \mathrm{~s}^{-1}\right)=3 \mu \mathrm{s}$ for the nascent blob in the edge region. However, this time increases dramatically $\left[\omega_{\mathrm{Z}}(0) \rightarrow 0\right]$ as 
the blob is launched. The divergence of $\tau_{\mathrm{ed}}$, and consequent vanishing of the charge mixing by spin, ${ }^{10}$ allows charge separation and triggers blob radial propulsion. Thus, the blob achieves coherency by radial motion rather than internal vorticity.

The blob's velocity is well approximated by the $\mathbf{E} \times \mathbf{B}$ drift. The electric field implied by the charge dipole has the proper orientation to propel the central region of the blob in the positive-x direction, i.e. radially toward the wall. This picture is repeated for all blobs examined and is consistent with the basic model of blob dynamics discussed above. Thus, near the nose and in a frame moving with the density maximum, the flow consists of two counter-rotating patches that allow the blob to move smoothly through the ambient SOL plasma, like a swimmer executing the breaststroke.

Some qualitative features in Fig. 2 are consistent with the simple $2 \mathrm{D}$ blob model: ${ }^{1,2}$ (i) the relatively fast decay of central vorticity compared to the dipole vorticity; (ii) the emergence of the vorticity (charge) dipole at $\mathrm{x}$ $\approx 1.5 \mathrm{~cm}$ coincident with the emission (radial propagation) of the blob; and (iii) the decrease in magnitude of the dipole vorticity as the blob propagates into the SOL, consistent with decreasing blob density from parallel losses and losses to the blob's tail. However, some quantitative discrepancies with the 2D model motivate the development of a 3D theory.

One can calculate $\mathrm{v}_{\mathrm{X}}=\mathrm{dx} / \mathrm{dt}$ both by finite differencing trajectories and from the $\mathbf{E} \times \mathbf{B}$ drift velocity $\mathrm{v}_{\mathrm{Ex}}$ at the density maximum. For several blobs examined in detail, the two velocities agree to within a factor of two and give values in the range $v_{X}=1-6 \mathrm{~km} / \mathrm{s}$, where $c_{s}=20-30 \mathrm{~km} / \mathrm{s}$ in the SOL. However, application of the 2D sheathconnected model predicts $\mathrm{v}_{\mathrm{Ex}}$ up to two orders of magnitude smaller for the present simulations.

To examine the observed velocity scaling with blob radius, $\mathrm{v}_{\mathrm{Ex}}(a)$, in detail, we projected the vorticity fluctuations at $\mathrm{x}=3 \mathrm{~cm}$ onto a set of wavelets of the form $\{\mathrm{w}\}=\left\{\sin \left[\pi\left(\mathrm{y}-\mathrm{y}_{0}\right) / 2 a\right]\right.$ if $|\mathrm{y}-\mathrm{y} 0| \leq 2 a, 0$ otherwise $\}$, with free parameters $\left\{\mathrm{y}_{0}, a\right\}$. The goodness of fit was defined as $(\mathrm{w} \cdot \mathrm{f}) / \sqrt{ }(\mathrm{w} \cdot \mathrm{w} \mathrm{f} \cdot \mathrm{f})$, with the dot product (integral in $\left.\mathrm{y}\right)$ restricted to the wavelet window, $\left|\mathrm{y}-\mathrm{y}_{0}\right| \leq 2 a$. A charge dipole oriented with the positive pole at greater $\mathrm{y}$ than the negative pole, corresponding to a blob drift in the positive-x direction, will register with positive goodness. The analysis was performed on all vorticity fluctuations for $\mathrm{t}>800 \mu$ s, i.e. not restricted to visually distinguishable 
blobs. We found that points $\left(\mathrm{y}_{0}, a\right)$ with goodness $>0.8$ have the following properties: (a) $\mathrm{v}_{\mathrm{Ex}}\left(\mathrm{y}_{0}\right)>0$ with statistically ignorable exceptions; (b) $\left\langle\mathrm{v}_{\mathrm{Ex}}\right\rangle_{\mathrm{y} 0}=1.0 \pm 0.5 \mathrm{~km} / \mathrm{s}$, independent of $a$, to within error bars; and (c) the distribution function $\mathrm{P}(a)$, is nearly independent of goodness and is dominated by small events $(a \sim 0.5 \mathrm{~cm}$, not prominent visually) with a significant tail distribution peaking at $a \sim 1.5 \mathrm{~cm}$, corresponding to the more visible blobs (e.g. Fig. 1). Although the range of $a$ obtained in the simulation is remarkably limited, the observed scaling $\mathrm{v}_{\mathrm{X}} \propto \mathrm{a}^{0}$, disagrees with the $1 / \mathrm{a}^{2}$ scaling that pertains in the sheath-connected limit. It is roughly consistent with the 3D model, which we will present.

The appearance of the blobs in the SOL coincides in time $(\mathrm{t}>800 \mu \mathrm{s})$ with the loss of correlations between $\phi$ (or $\mathrm{n}$ ) at the sheath and at the outboard midplane, as shown in Fig. 3. As we shall see, the mathematical structure governing the nonlinear dynamics changes as well.

The magnitude and internal structure of the blob temperature $\mathrm{T}_{\mathrm{e}}$ provides additional evidence for blob disconnection from the divertor sheath. We observe that the temperature fluctuations are as large as the mean temperature in the SOL, are well correlated with potential fluctuations in the SOL (and in the edge) and form a dipole that is in phase with the vorticity and potential dipoles associated with the blobs.

These observations can be explained by a 3D theory of disconnected blobs ${ }^{11}$ extended here to include parallel electron heat flow, X-point cooling due to neutral fueling and ion polarization currents. In this picture, the curvature drift drives charge polarization of the blob, as in 2D theory, resulting in a dipole distribution of charge, potential and vorticity. However, $\mathrm{J}_{\|}$is prevented from flowing to the sheaths by the large parallel plasma resistivity $\eta_{\|}$in the divertor region due to strong cooling associated with neutral fueling. Instead, the + and charged regions are "short-circuited" by current flowing across the thin elliptical fanned regions at the X-points. This effectively replaces the sheath boundary condition by an X-point boundary condition. ${ }^{11}$ Any of several mechanisms (electron-ion collisions, ion viscosity, ion polarization drifts) can provide the $\mathbf{J}_{\perp}$ that closes the blob current loop and disconnects it from the sheaths.

In Braginskii collisional fluid theory, a thermoelectric force produces the temperature dipole $\left(\nabla_{\|} \mathrm{T}_{\mathrm{e}} \neq 0\right)$ when $\nabla_{\|} \phi \neq 0$, as illustrated by the parallel Ohm's Law, with $\nabla_{\|} \mathrm{n}=0$ : 


$$
\eta_{\|} \mathrm{J}_{\|}+\nabla_{\|} \phi=1.71 \nabla_{\|} \mathrm{T}_{\mathrm{e}} / \mathrm{e}
$$

Deep into the $\mathrm{X}$-point region $\mathrm{J}_{\|}, \nabla_{\|} \phi$ and $\nabla_{\|} \mathrm{T}_{\mathrm{e}}$ must vanish for a disconnected solution by assumption, permitting a linearized solution for the coupled vorticity, Ohm's law and parallel electron heat flow equations. In the eikonal limit, one can obtain an explicit solution with an exponentially-decaying current, $\mathrm{J}_{\|}(\mathrm{z})=\mathrm{J}_{\| \mathrm{X}} \mathrm{e}^{-\gamma \mathrm{z}}, \gamma \sim\left(\sigma_{\perp} / \sigma_{\|}\right)^{1 / 2}$ $a^{-1}$, which is disconnected from the sheath if $\gamma \mathrm{L}_{\|} \gg 1$. Here, $\mathrm{L}_{\|}$is the field line length, $\sigma_{\|}$and $\sigma_{\perp}$ are the conductivities parallel and perpendicular to $\mathbf{B}$, and $\mathrm{J}_{\| \mathrm{X}}$ is the current density at the entrance to the $\mathrm{X}$-point region. This solution has the property that the total parallel heat flux vanishes, i.e. the heat conduction $\left(-\kappa_{\|} \nabla_{\|} \mathrm{T}_{\mathrm{e}}\right)$ and convection $\left(\propto-\mathrm{J}_{\|} \mathrm{T}_{\mathrm{e}}\right)$ terms balance. The resulting $\mathrm{J}_{\|}$is in the directions of $-\nabla_{\|} \phi$ and $-\nabla_{\|} \mathrm{T}_{\mathrm{e}}$ so that $\mathrm{J}_{\|}$has the correct sign to (i) reduce the charge polarization, and (ii) carry electron heat flux up the parallel temperature gradient, allowing the temperature dipole to persist. Thus, the $\mathrm{T}_{\mathrm{e}}, \mathrm{J}_{\|}$and $\phi$ dipoles are all in phase at the midplane, as observed in the simulations. Because the fluctuations are order unity and the present version of the code employs toroidally averaged plasma parameters in the Braginskii coefficients, e.g. $\kappa_{\|}, \eta_{\|}$, there is nothing to prevent small local negative temperature excursions in the simulations. In fact these are occasionally observed, but in light of the preceding discussion their presence seems benign.

The eikonal, fully disconnected X-point boundary condition for blobs is of the form ${ }^{11}$

$$
\mathrm{J}_{\|}\left( \pm \mathrm{L}_{\|}\right)= \pm \mathrm{k}_{\perp}\left(\sigma_{\perp} \sigma_{\|}\right)^{1 / 2} \phi \equiv \alpha \phi
$$

where $\alpha$ is an "effective conductivity" parameter. This parallel current drains the charge imbalance driven by the curvature drifts, and provides a blob "voltage-current" relation that determines $\phi$ and $\mathrm{v}_{\mathrm{EX}_{\mathrm{X}}}$.

To describe the simulations, we take $\sigma_{\|}$to be the usual collisional result $\left(\sigma_{\|}=2 \mathrm{ne}^{2} / \mathrm{m}_{\mathrm{e}} v_{\mathrm{e}}\right)$ and compare various mechanisms for $\sigma_{\perp}$. Estimates show that the (nonlinear) ion polarization current gives the largest contribution in the X-point region with $\left(4 \pi \mathrm{v}_{\mathrm{A}}{ }^{2 / \mathrm{c}^{2}}\right) \nabla \cdot \mathbf{J}_{\perp \text { pol }} \approx-(\mathrm{c} / \mathrm{B}) \mathbf{b} \times \nabla \phi \cdot \nabla \nabla^{2} \phi \sim(\mathrm{c} / \mathrm{B}) \phi^{2} / a^{4}$. As a heuristic procedure for order-of-magnitude estimates of this nonlinear term, we write $\mathbf{J}_{\perp \text { pol }} \equiv-\sigma_{\perp \text { pol }} \nabla_{\perp} \phi \sim \sigma_{\perp \text { pol }} \phi / a$. It follows that $\sigma_{\perp \text { pol }}=\left(\mathrm{c}^{2} \Omega_{\mathrm{i}} / 4 \pi \mathrm{v}_{\mathrm{A}}{ }^{2}\right)\left(\mathrm{e} \phi / \mathrm{T}_{\mathrm{e}}\right)\left(\rho_{\mathrm{s}} / a\right)^{2} \propto \phi / a^{2}$, where $\mathrm{v}_{\mathrm{A}}$ is the Alfvén velocity, $\Omega_{\mathrm{i}}$ is the cyclotron 
frequency and $\rho_{\mathrm{s}}=\mathrm{c}_{\mathrm{S}} / \Omega_{\mathrm{i}}$. Substituting $\sigma_{\|}$and $\sigma_{\perp \text { pol }}$ into Eq. (2), gives $\mathrm{J}_{\|}$at the X-points in terms of $\phi$ and $a$. Balancing this current loss term with the curvature source term in the midplane vorticity equation, $\nabla_{\|} \mathbf{J}_{\|}=-\nabla_{\perp} \cdot \mathbf{J}_{\kappa}$ and using $\nabla_{\perp} \cdot \mathbf{J}_{\kappa}=\left(2 \mathrm{c} / \mathrm{B}^{2}\right) \mathbf{B} \times \boldsymbol{\kappa} \cdot \nabla \mathrm{p} \sim \operatorname{nec}_{\mathrm{S}}\left(\rho_{\mathrm{S}} / a \mathrm{R}\right)$ with $\boldsymbol{\kappa} \sim-\mathbf{e}_{\mathrm{X}} / \mathrm{R}\left(\mathrm{R}=\right.$ major radius), gives e $\phi / \mathrm{T}_{\mathrm{e}} \sim$ $\left(v_{\mathrm{e}} / \Omega_{\mathrm{e}}\right)^{1 / 3}\left(a \mathrm{~L} \| / \rho_{\mathrm{S}} \mathrm{R}\right)^{2 / 3}$ and $\mathrm{v}_{\mathrm{Ex}} \sim \mathrm{c}_{\mathrm{S}}\left(\mathrm{e} \phi / \mathrm{T}_{\mathrm{e}}\right)\left(\rho_{\mathrm{S}} / a\right)$. For typical simulation parameters, $\mathrm{e} \phi / \mathrm{T}_{\mathrm{e}} \sim \mathrm{O}(1)$, as observed, and $\mathrm{v}_{\mathrm{Ex}}$ from this $3 \mathrm{D}$ disconnected blob model correctly estimates blob speeds. Furthermore, $\mathrm{v}_{\mathrm{Ex}} \propto a^{-1 / 3}$, which is consistent, within the error bars, with the simulation result $\mathrm{v}_{\mathrm{Ex}}(a) \sim \mathrm{a}^{0}$.

One can represent this physics by an equivalent circuit with a fixed current source $\mathbf{J}_{\kappa}$ (curvature-drift) and effective resistances for charge flow parallel $\left(R_{\|}\right)$and perpendicular $\left(R_{\perp}\right)$ to $B$ and across the sheaths $\left(R_{s h}\right)$. Increasing $\mathrm{R}_{\|}$in the $\mathrm{X}$-point and divertor (by neutral cooling) causes the current to flow across $\mathbf{B}$, increasing the total resistance of the circuit. A larger voltage $(\phi)$ is then required to maintain the steady-state current. Thus, the conditions that a blob disconnects from the sheaths and moves faster radially $\left(v_{E x} \propto \phi\right)$ are $R_{s h}<<R_{\perp}<<R_{\|}$. The details will be presented elsewhere.

In summary, the simulations and model show that: (1) blobs arise in the nonlinear stages of turbulence and propagate outwards; (2) the curvature drift drives the charge dipole and the corresponding $\mathbf{E} \times \mathbf{B}$ drift $\mathbf{v}_{\mathrm{Ex}}$, as in the $2 \mathrm{D}$ theory, but $\mathrm{v}_{\mathrm{Ex}}$ is larger in the $3 \mathrm{D}$ theory because the blob voltage-current relation is modified and results in $\mathrm{e} \phi / \mathrm{T}_{\mathrm{e}} \sim 1$; (3) $\mathrm{J}_{\|}$disconnects from the sheaths because of the large $\eta_{\|}$(due to neutral-induced cooling) and large $\sigma_{\perp}$ (due to the elliptical distortions of the flux tubes) in the X-point region; (4) the X-point "short-circuiting" of $\mathrm{J}_{\|}$ establishes a temperature dipole in phase with the charge and vorticity dipoles; (5) blob disconnection by ion polarization drifts is related to the physics of the resistive X-point (RX) mode turbulence ${ }^{12}$ which describes the linear phase of the simulations. ${ }^{7}$ Thus, the present work has interesting implications for edge and SOL transport: in cool, highly-collisional divertor plasmas the blobs become disconnected from the sheaths and their radial velocity (and associated transport) increases. This may provide a convective mechanism for the density limit, as suggested by data from Alcator C-MOD. ${ }^{13}$ 
Nonlinear coherent structures have previously been observed in 2D simulations of tokamak edge plasmas. ${ }^{14,15}$ Here we have shown that similar structures also emerge from strong 3D plasma turbulence, with significant differences. Parallel and perpendicular processes interact to determine the dynamics of these objects, but their transport properties can be understood from relatively simple models. In some important regimes these coherent objects can govern the cross-field transport of plasma in the tokamak edge region.

\section{Acknowledgements}

We thank S. Krasheninnikov and D. Ryutov for useful conversations. This work was supported by US DOE grant DE-FG03-97ER54392 and LLNL contract \# W-7405-Eng-48.

\section{References}

${ }^{1}$ S. I. Krasheninnikov, Phys. Lett. A 283, 368 (2001).

${ }^{2}$ D. A. D’Ippolito, J. R. Myra, and S. I. Krasheninnikov, Phys. Plasmas 9, 222 (2002).

${ }^{3}$ S. J. Zweben, et al., Nucl. Fusion 44, 134 (2004); G. Y. Antar, et al Phys. Plasmas 10, 419 (2001); J. A. Boedo, et al, Phys. Plasmas 8, 4826 (2001).

${ }^{4}$ P. B. Parks, W. D. Sessions, and L. R. Baylor, Phys. Plasmas 7, 1968 (2000).

${ }^{5}$ A short review is given in: D. A. D'Ippolito, J. R. Myra, S. I. Krasheninnikov, G. Q. Yu, and A. Yu. Pigarov, Contrib. Plasma Phys 44, 205 (2004).

${ }^{6}$ X. Q. Xu et al., Bull. Am. Phys. Soc. 48, 184 (2003), paper KP1-20.

${ }^{7}$ X. Q. Xu et al., New Journal Phys. 4, 53.1 (2002).

${ }^{8}$ X. Q. Xu et al., Contrib. Plasma Physics 44, 105 (2004).

${ }^{9}$ W. M. Nevins, GKV manual in preparation (2004).

${ }^{10}$ J. R. Myra, D. A. D'Ippolito, S. I. Krasheninnikov, and G. Q. Yu, to be published in Phys. Plasmas 11, (2004).

${ }^{11}$ D. D. Ryutov and R. H. Cohen, Contrib. Plasma Phys 44, 111 (2004); S. I. Krasheninnikov, D. D. Ryutov, and G. Q. Yu, J. Plasma and Fus. Research 6 (2004), to appear.

12 J. R. Myra, D. A. D'Ippolito, X. Q. Xu, and R. H. Cohen, Contrib. Plasma Phys. 40, 352 (2000). 
${ }^{13}$ M. Greenwald, Plasma Phys. Contr. Fusion 44, R27 (2002).

${ }^{14}$ Y. Sarazin and Ph. Ghendrih, Phys. Plasmas 5, 4214 (1998).

${ }^{15}$ O. E. Garcia, V. Naulin, A. H. Nielsen, and J. Juul Rasmussen, Phys. Rev. Lett. 92, 165003 (2004). 
Figure 1

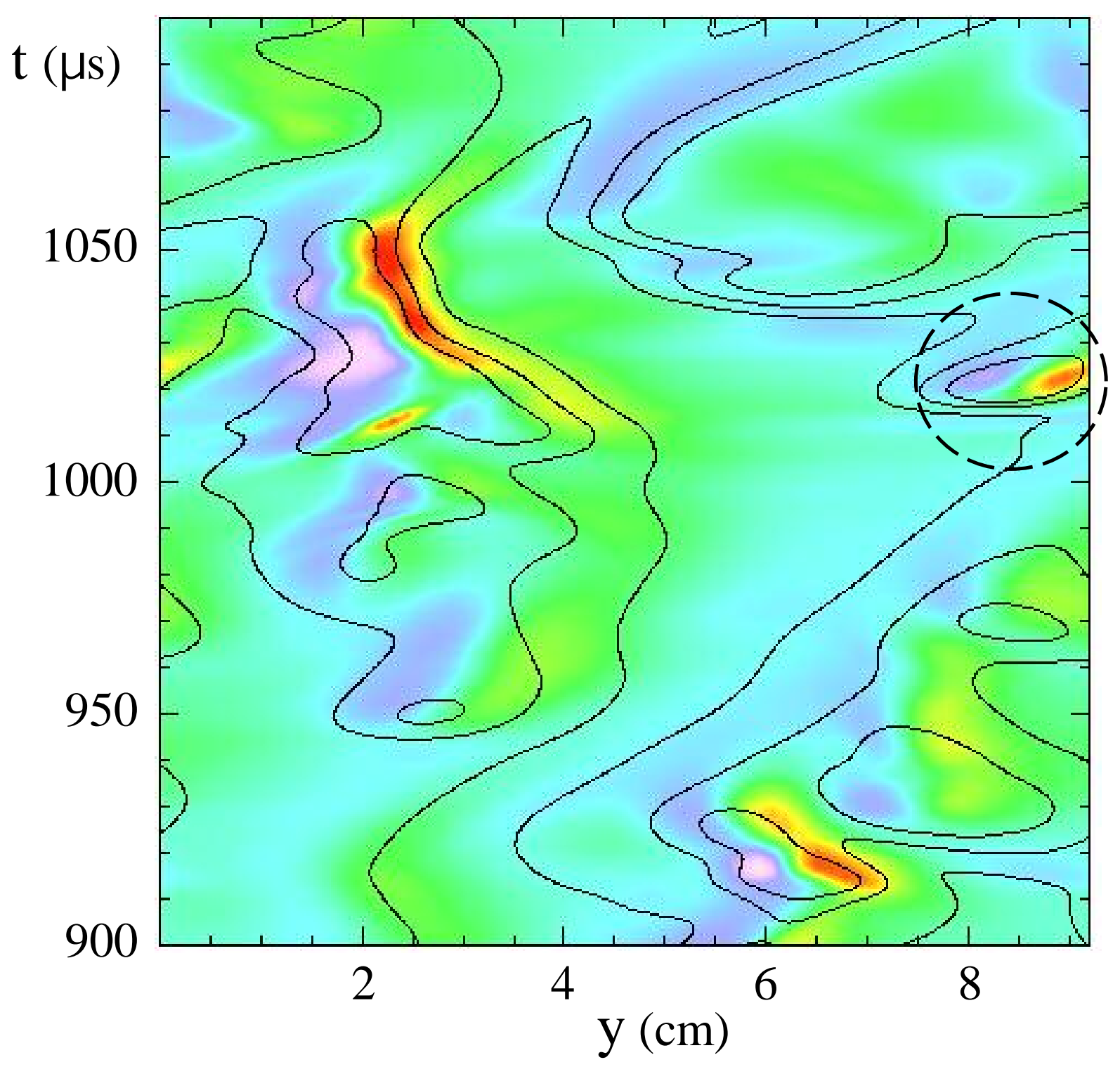


Figure 2

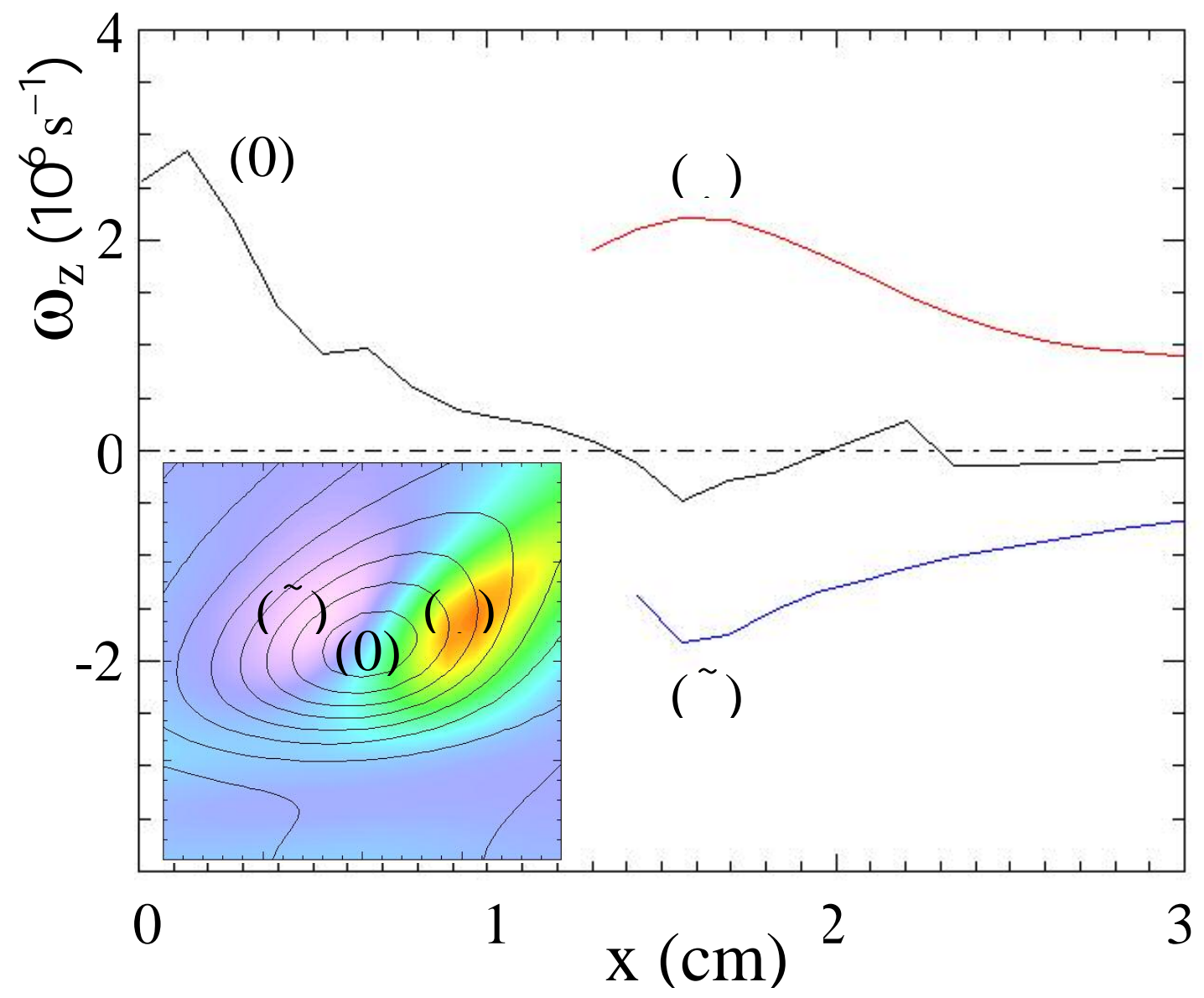


Figure 3

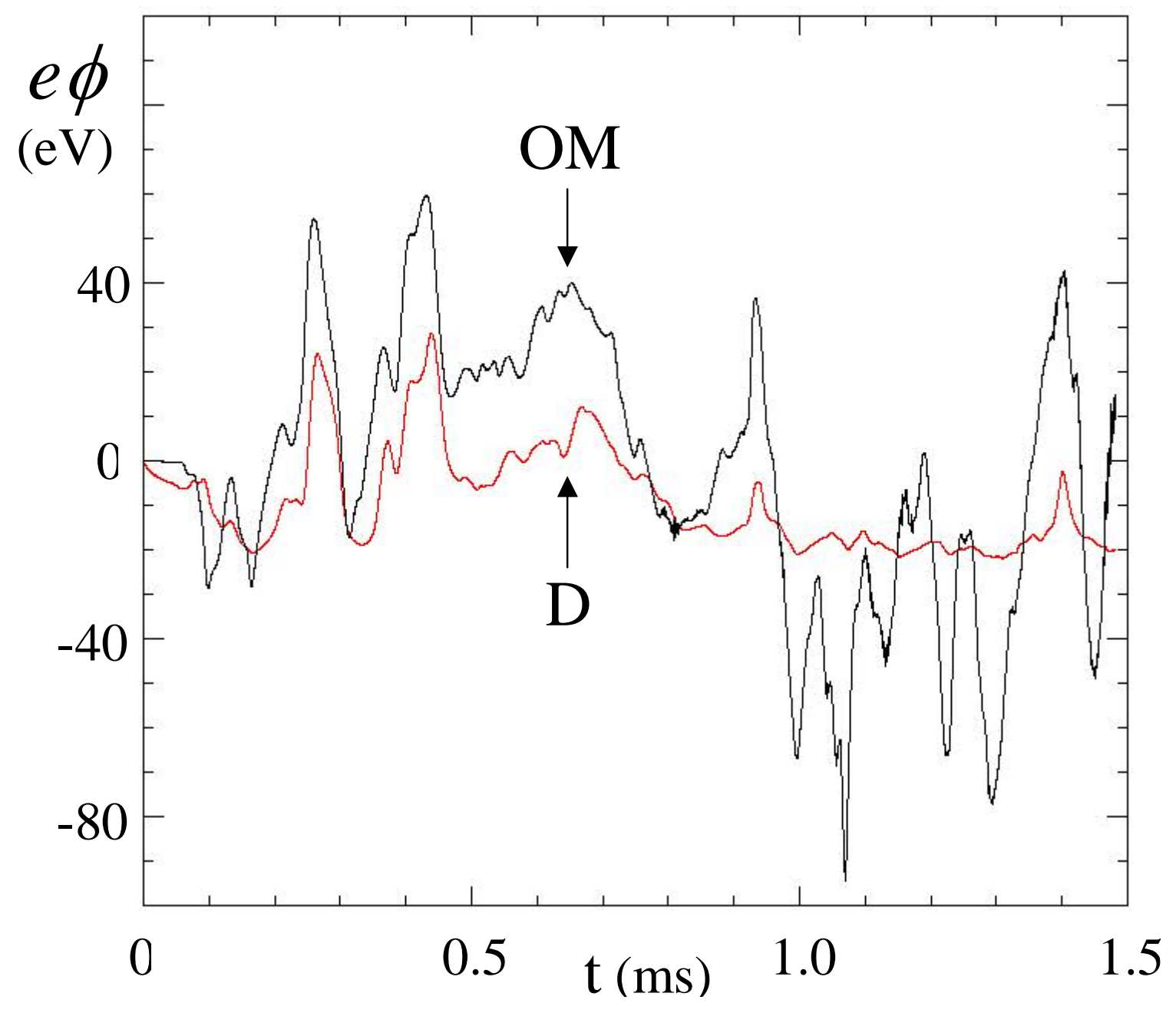




\section{Figure Captions}

(1) Ion density contour lines superimposed on an intensity plot of charge density (red: positive, blue: negative).

(2) Maximum (+) and minimum (-) vorticity and vorticity at density maximum (0) vs. radial position of the blob circled in Figure 1, enlarged in the inset.

(3) Electrostatic potential at a point in the outboard midplane (OM) and at the corresponding point in the divertor sheath (D). Note the apparent disconnection for $t>800 \mu \mathrm{s}$. 